\title{
Characterisation of Herschel-SPIRE flight model optical performances
}

Marc Ferlet, Glenn Laurent, Bruce Swinyard, Jason Glenn, Jamie Bock, et al.

Marc Ferlet, Glenn Laurent, Bruce Swinyard, Jason Glenn, Jamie Bock, Kjetil Dohlen, "Characterisation of Herschel-SPIRE flight model optical performances," Proc. SPIE 7010, Space Telescopes and Instrumentation 2008: Optical, Infrared, and Millimeter, 70102 U (12 July 2008); doi: $10.1117 / 12.788888$

EDIE Event: SPIE Astronomical Telescopes + Instrumentation, 2008, Marseille, France 


\title{
Characterisation of Herschel-SPIRE flight model optical performances
}

\author{
Marc Ferlet ${ }^{* a}$; Glenn Laurent ${ }^{\mathrm{b}}$; Bruce Swinyard ${ }^{\mathrm{a}}$; Jason Glenn ${ }^{\mathrm{b}}$; Jamie Bock ${ }^{\mathrm{c}, \mathrm{d}} ;$ Kjetil Dohlen $^{\mathrm{e}}$

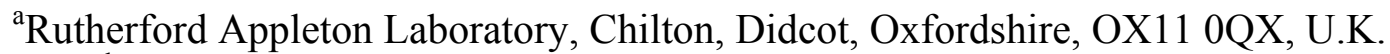 \\ ${ }^{\mathrm{b}}$ University of Colorado, CASA 389-UCB, Boulder, CO 80309, U.S.A. \\ ${ }^{\mathrm{c} J e t}$ Propulsion Laboratory, Pasadena, CA 91109, U.S.A.

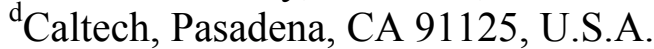 \\ e Observatoire Astronomique de Marseille Provence, 2 Place Leverrier, 13248 Marseille, France
}

\begin{abstract}
The Spectral and Photometric Imaging Receiver (SPIRE) is one of three scientific instruments on ESA's Herschel Space Observatory. This long wavelength instrument covers 200 to $670 \mu \mathrm{m}$ with a three band photometric camera and a two band imaging Fourier Transform Spectrometer (IFTS). Following first results reported in a previous paper, we discuss the in-band optical performances of the flight model as measured extensively during several dedicated test campaigns. Complementary to the experimentally probed spectral characteristics of the instrument detailed in an accompanying paper (see L.D. Spencer et al., in these proceedings), attention is focused here on a set of standard but key tests aimed at measuring the spatial response of the Photometer and Spectrometer end-to-end optical chain, including detector. Effects of defocus as well as source size extent, in-band wavelength, and polarization are also investigated over respective Photometer and Spectrometer field-of-views. Comparison with optical modelling, based on instrument design knowledge and some of the internal component measured characteristics, is performed. Beyond the specific characterisation of each effect, this allows estimating in each band where optical behaviour and detector behaviour respectively dominates and also reconstructing some of the contributors to the instrument throughput. Based on this analysis, retrieved optical performances are finally assessed against the related science-driven instrument requirements.
\end{abstract}

Keywords: Herschel, SPIRE, far-infrared, sub-millimetre, optical characterisation, performances verification

\section{INTRODUCTION}

SPIRE $^{[1]}$, the Spectral and Photometric Imaging Receiver, is one of three focal plane instruments on the ESA/NASA Herschel Space Observatory (HSO). The primary mirror of Herschel is $3.5 \mathrm{~m}$ in diameter and is passively cooled to a temperature of $\sim 80 \mathrm{~K}$. The SPIRE instrument consists of two separate sub-instruments: a three-band Photometer and a two-band imaging Fourier transform spectrometer ${ }^{[2]}$ (IFTS) covering the wavelength region from $200-670 \mu \mathrm{m}$. The Photometer has an 8x4arcmin field of view and uses dichroics to allow simultaneous observations of the field in three bands. Similarly, the IFTS has a 2.6arcmin field-of-view (FoV), separated from the Photometer field. The bolometric detectors, arranged in arrays displayed in Figure 1 below, employ smooth walled feedhorns ${ }^{[3]}$, with $2 \mathrm{~F} \lambda_{\mathrm{o}}$ aperture, with waveguides designed to give single mode beams over the bandpass in the photometer and are multi-moded beams in the Spectrometer. The waveguide cut-offs control the long wavelength edge of each bandpass with the short wavelength edges controlled by the filtering scheme.

In the following subsections, we recall first the key elements of the SPIRE optical design ${ }^{[4]}$ and then the main elements of the test facility used through the instrument level test (ILT) 5 campaigns for the proto-flight model (PFM), from PFM1 to PFM5. First results of the partially populated SPIRE PFM focal plane unit (FPU), related to PFM1 and 2 test campaigns have been previously reported ${ }^{[5]}$. PFM5 was done under completely closed cryostat with darker and more stable background environment for further detector characterisation and so no external source for optical characterisation was used. As a final follow-up of a previous report ${ }^{[6]}$ on the optical performances and complementary to the specific

${ }^{*}$ Contact details: email: M.Ferlet@rl.ac.uk; phone: +(44/0) 1235446409

Space Telescopes and Instrumentation 2008: Optical, Infrared, and Millimeter, edited by Jacobus M. Oschmann, Jr., Mattheus W. M. de Graauw, Howard A. MacEwen, Proc. of SPIE Vol. 7010, 70102U, (2008) · 0277-786X/08/\$18 · doi: 10.1117/12.788888 
spectrometric performances evaluation ${ }^{[7]}$ of the IFTS, the subsequent main sections of this paper focus on the spatial performances of both Photometer and Spectrometer during PFM3 and, post final vibrations test, during PFM4.

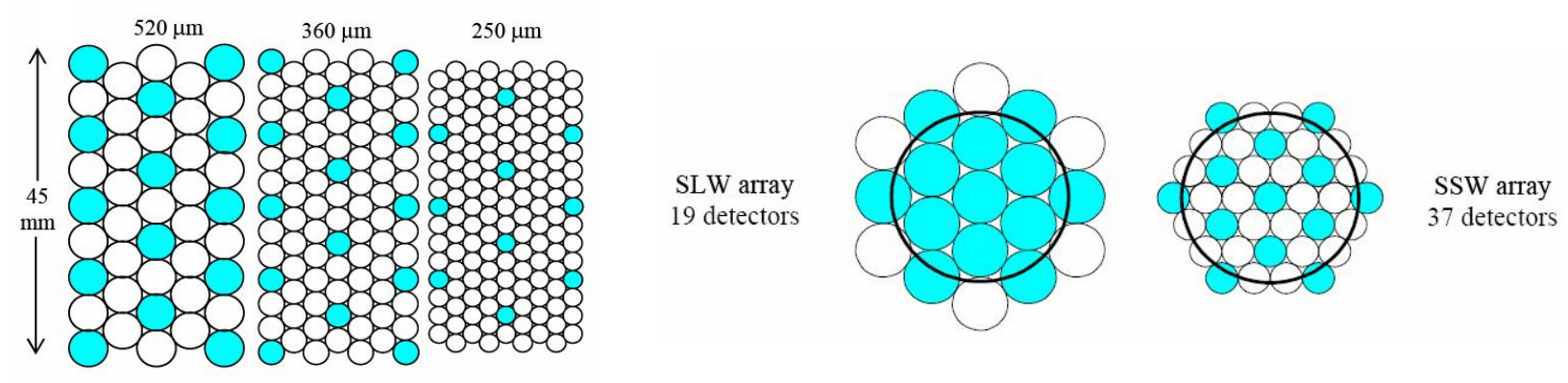

Figure 1: Layout of the Photometer arrays, PLW, PMW and PSW (left) and Spectrometer detector arrays, SLW and SSW (right). The blue-shaded detector pixels are nominally co-aligned on the sky in the respective Photometer and Spectrometer bands.

\subsection{Optical design}

The first mirror in the SPIRE optical chain, denoted M3, is an off-axis ellipsoid projecting an image of the telescope secondary (M2) onto M4 (=Beam Steering Mirror or BSM) where we place a steerable flat mirror whose orientation is adjustable in flight to permit $\pm 2^{\prime}$ chopping in the sagittal plane, allowing off-field chopping for a $4^{\prime} \times 4^{\prime}$ sub-field, and $\pm 30^{\prime \prime}$ beam steering motion in both the tangential and sagittal planes to obtain fully Nyquist sampled images. The image of M2 is well-corrected and in focus at the centre of M4 so that the pupil image at the cold stop stays fixed during chopping and beam steering. The missing obscured part of the pupil image at M4 is filled with a calibration source PCAL. The toric M5 mirror re-images the focal plane onto a field mirror at M6. While M3 and M4 are common for both photometer and spectrometer, the two systems separate at M6. The photometer M6 is toric and sends the beam into an Offner-type relay system consisting of three spherical mirrors: M7 (concave), M8 (convex), and M9 (concave). Tilt angles, separations and curvatures of these mirrors provide enough free variables to give an easily accessible pupil image between M8 and M9 in which the cold stop is located. At this point there is also a physical separation between the $\sim 4 \mathrm{~K}$ structure surrounding the entire instrument and the sub-2K structure, or cold box, to which the detectors are bolted. The optical relay also gives a system with a long back focal length allowing the three wavelength channels (PSW, PMW and PLW) to be separated by a pair of high-pass dichroics, PDic1 and PDic2.
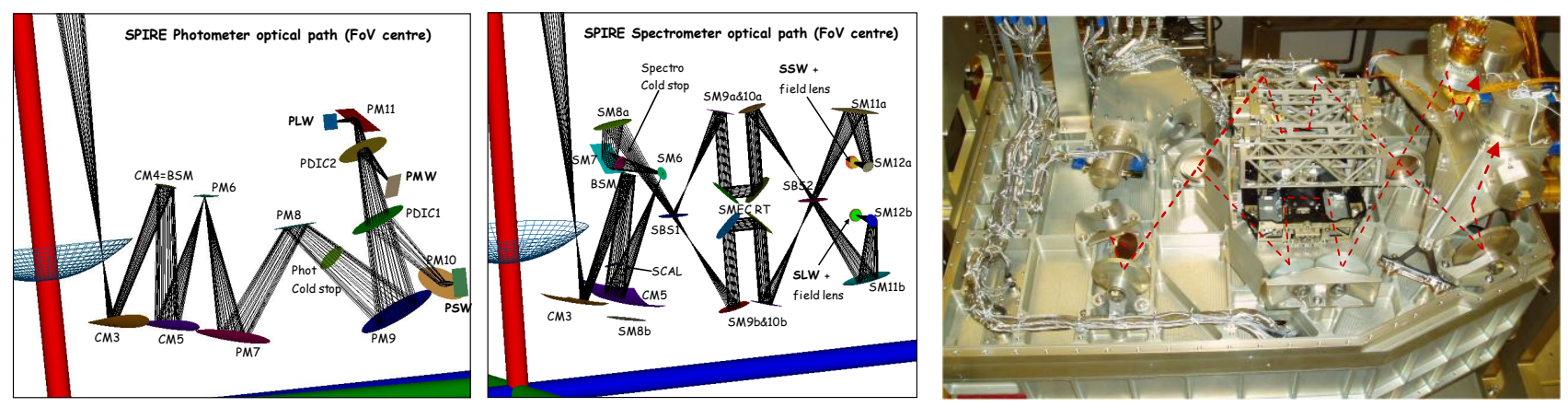

Figure 2: Optical layout with FoV centre raytrace for the Photometer (left) and Spectrometer (centre). Fully integrated SPIRE FPU Flight Model with its cover removed to allow view access to the Spectrometer side (right).

After reflection from the common mirrors M3, M4, and M5, the spectrometer beam is picked off by the toric M6 and sent out of the plane of the photometer system. The flat M7 redirects it into a plane parallel to the photometer optical chain but separated from it by $170 \mathrm{~mm}$. The two parts of the instrument are thus laid out physically on two sides of a common optical bench structure making the SPIRE FPU structure well optimised in terms of mass. The input relay mirror (SM8) focuses the beam to an intermediate image plane located just after the first beam splitter, after which the beam is collimated (SM9) and sent vertically towards the roof top mirror (RT) on the spectrometer mechanism. The rooftop shifts the beam and sends it up towards the camera mirror (SM10). The camera is symmetrical with the collimator, and focuses the beam to an image plane just before the output beam splitter. The output relay mirror (SM11) 
in turn focuses the beam onto the detector arrays, after going through additional field lenses included to improve telecentricity and coupling with the detector pixels at Spectrometer FoV edge. To accommodate the components within the available volume, a fold mirror is needed to take the beam out of the plane again. A pupil image is located near the final fold mirror, making this a convenient place for the entrance hole in the $2 \mathrm{~K}$ enclosure. This pupil moves as the OPD changes, however, so it is not appropriate for a limiting cold stop. Instead, a limiting aperture is placed in another pupil image at $4 \mathrm{~K}$ located between M6 and M7. The other half is symmetrical in reflection through the line of the two beam splitters; the only difference being that the fold mirror M7 is absent and a second calibration source (SCAL) is placed at the image of the pupil.

\subsection{Dedicated test set-up}

The test environment for instrument level testing at the Rutherford Appleton Laboratory consists of a Helium cryostat that provides the correct temperature levels for the FPU and an external (warm) telescope simulator and sources ${ }^{[8]}$. In order to replicate the $\mathrm{f} / 8.68$ beam of the HSO telescope, we use a 1:1 imaging system based on an off-axis ellipsoidal mirror, a scaled-down pupil aperture mask replicating the HSO telescope pupil obscuration, and a system of flat folding mirrors that allow the input focus to be moved around the SPIRE FoV whilst maintaining focus. Defocusing, decoupled from FoV scanning, is possible independently over a large axial range. Collectively this system is known as the telescope simulator. The telescope simulator can be fed by one of two separate sources - a molecular laser giving monochromatic lines in the $30-1000 \mu \mathrm{m}$ range and a hot blackbody (HBB) that, in turn, is seen through a Michelson interferometer to allow the photometer bandpasses to be evaluated.

Using the re-pointing capability of the telescope simulator to reach any pixel in the SPRIE combined FoV and its scanning ability, raster-type 2D beamscans or maps of the individual focal plane pixel beam pattern can be measured. When done at best focus and with very small source size extent (point source case), the characteristics spatial impulse function or PSF of SPIRE optics and detector can be obtained. Coupled with broadband source, it allows full-band spatial response characterisation to continuum source. With a tuneable monochromatic source such as the FIR laser, one can get an insight on the in-band spectral variations of the PSF and derived quantities. Sections 2, 3 and 4 below report on the results of such tests for the Photometer and Spectrometer, while section 5 discusses the impact of defocusing the test beam and the response to varying source size and polarisation state.

\section{PHOTOMETER SPATIAL RESPONSE CHARACTERISATION}

Summarised in the graph below are the measured beamsizes, in terms of FWHM, of the broadband and monochromatic beam patterns for sets of pixels from the 3 Photometer arrays. These were generated after removal of the source size broadening effect, as discussed in the previous paper, when it was not possible to directly measure in point source conditions i.e. too low source signal.
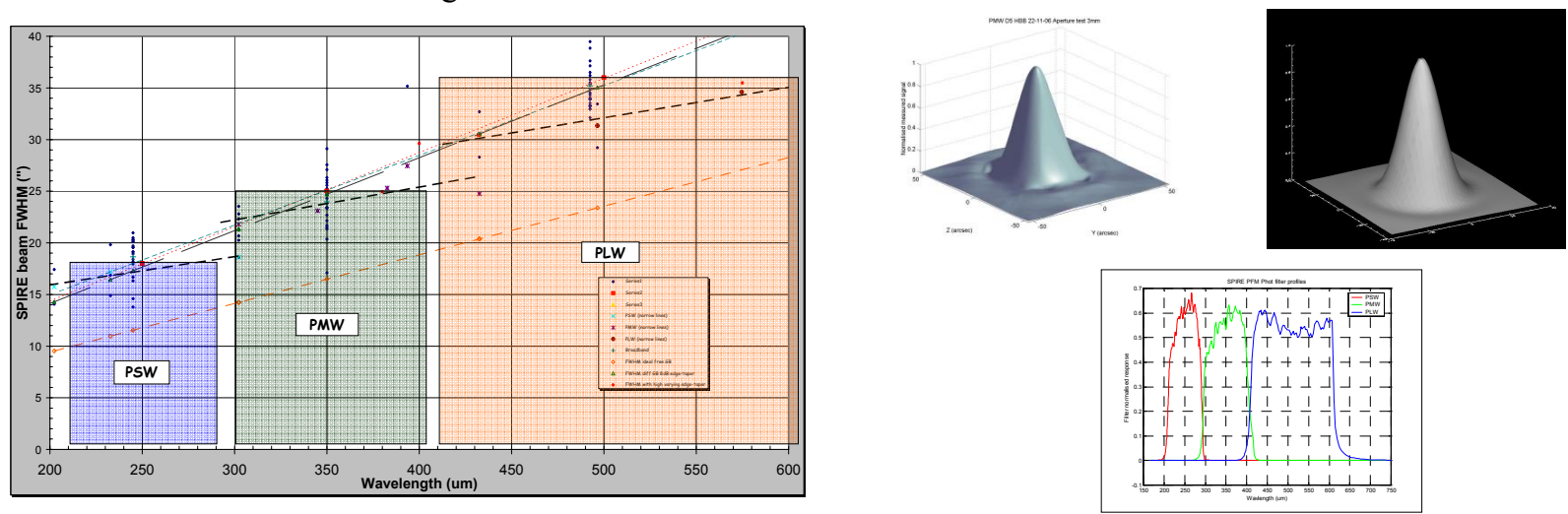

Figure 3: Summary of Photometer beamsize results (left), example of measured beam pattern on a PMW pixel and associated reconstruction by optical modelling for calibration file input, with the Photometer bandpasses from the filter measurements (right) 
The beam shape factor was found very close to Gaussian in most cases, in particular for the broadband source case. The spectral variations of the beam pattern in-band (dashed line in Figure 3 left) were found somewhat not increasing with wavelength as quickly as first order diffraction would predict. This was further analysed ${ }^{[9]}$ and explained by the measurements being actually performed at "best focus" respectively and by the low Fresnel number of the optical beam and its spectral dependence, increasing the effect at longer wavelength e.g. particularly PLW here.

All this was taken into account in the reconstruction of the broadband beam pattern for each array by optical modelling, leading to the final Photometer beamsize values below, suggested for calibration file inputs, for the typical cases of flat end-to-end spectrum and Rayleigh-Jeans (RJ) one:

\begin{tabular}{|c|c|c|c|}
\hline $\begin{array}{l}\text { Reconstructed broadband beam } \\
\text { profiles for SPIRE Photometer }\end{array}$ & PSW & PMW & PLW \\
\hline FWHM in case of flat spectrum & 18.8” & 24.6” & $35.6^{\prime \prime}$ \\
\hline FWHM in case of RJ spectrum & 18.5” & 24.3" & $34.9 "$ \\
\hline
\end{tabular}

These have all been found within 1" of the baseline beamsize requirements for the Photometer.

Alongside the filter stack transmission, the etendue $A \Omega$ is a key contributor to the optical chain throughput. Based on a formalism previously discussed ${ }^{[6]}$ and linked to the characteristics of the measured spatial response, spectral variations of the etendue are retrieved. Displayed in Figure 4, it shows a monotonic increase with wavelength in-band for PSW and PMW as expected, with higher values than pure $\lambda^{2}$ (which is limited to unapertured pure single mode Gaussian) due to diffraction angular broadening at limiting aperture such as cold stop. But for PLW a much flatter etendue is found and is thought to be linked to the noticed increase in relative PLW bandwidth due to shortwave shift of the PDic2 dichroic edge, dominating the cut-on of PLW bandpass ${ }^{[1]}$. As a consequence, the detector coupling elements (feedhorn+waveguide) are able to collect a relatively more important fraction of unfiltered in-band short wave through the second guided mode $\left(\mathrm{TM}_{01}\right)$ of this structure ${ }^{[3]}$. It generates increased acceptance efficiency by allowing coupling in this second mode as well while PSW and PMW, with narrower relative bandwidth, are limited to detection mainly in their respective $\mathrm{TE}_{11}$ first mode.
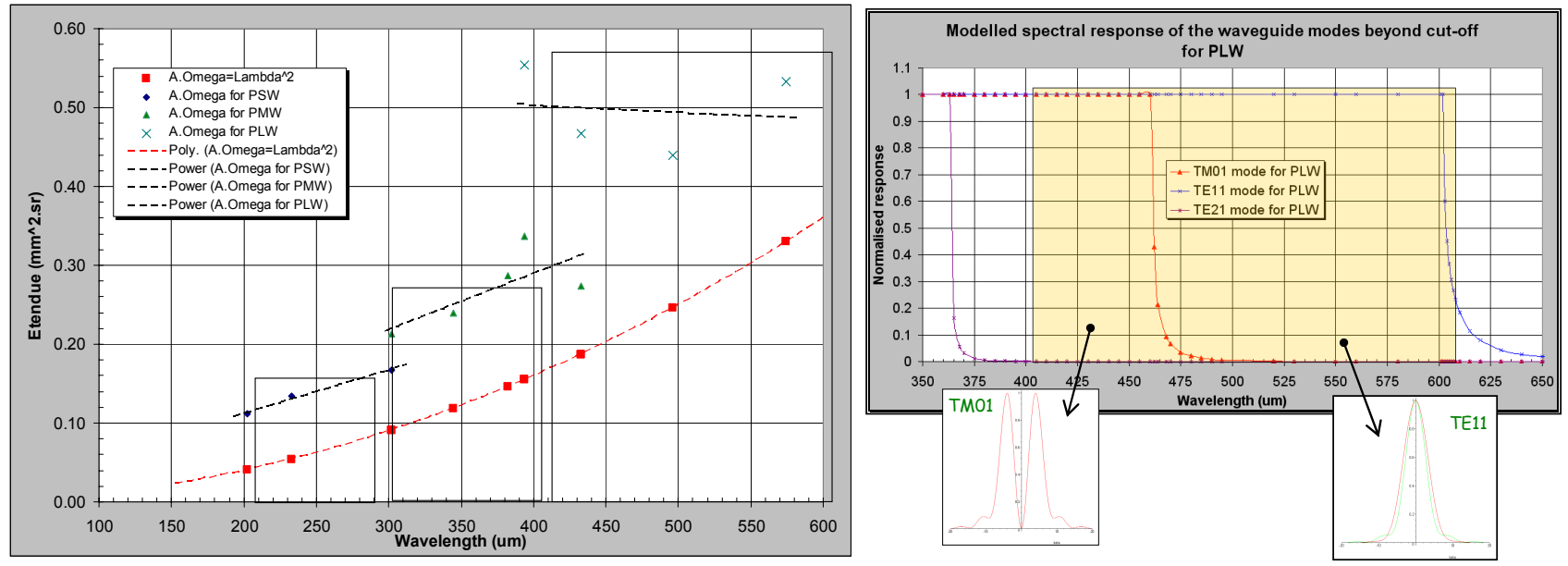

Figure 4: Retrieved Photometer etendue $A \Omega$ (left), and spectral variation and position of PLW first few modes cut-offs (right)

Using Wolf's theory of partial coherence and the associated coherent-mode decomposition of the cross-spectral density ${ }^{[10]}$, applied here in the Gauss-Shell model justified by the measured beam shape, the fractional power in the generalised fundamental mode (which is Gaussian) is obtained and displayed in Figure 5; along with indicative relative error on the reconstructed weighted sum of the mode coefficients from the numerically-truncated in practice mode decomposition. It gives a direct indication of the coupling efficiency, another contributor to the end-to-end throughput, when the source is single moded/spatially coherent. It provides an alternative approach used for validation of the coupling estimates previously derived ${ }^{[6]}$. For the case of wide spectrum (i.e. larger than the instrument bandpasses) source, the spectrum-weighted spectral average of the curve below must be taken and further confirm early estimates ${ }^{[6]}$. 


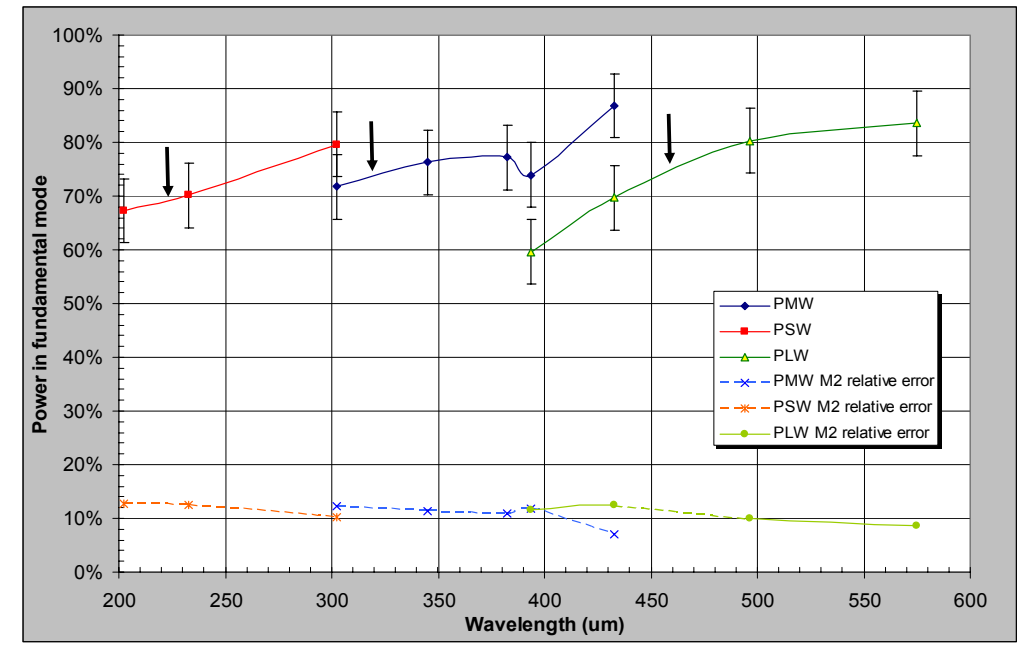

Figure 5: Spectral distribution of the fundamental mode relative power from the measured beam pattern modal decomposition in the Photometer bands. Additionally, black arrows indicate the spectral location of the respective mode cut-offs.

\section{SPECTROMETER SPATIAL RESPONSE CHARACTERISATION}

Similarly, the beamsizes from the measured Spectrometer beam patterns are reproduced below. For the case of thermal broadband source (HBB), the SSW average beamsize value reported at $250 \mu \mathrm{m}$ is found to be $17.3 \pm 1.5$ " while for SLW, reported at $350 \mu \mathrm{m}$, this reaches $25.8 \pm 1.7$ "; although in this last case, larger variations have been noticed depending on the exact source size (triggering further investigations discussed in 5.2). For the spectral lines case, both bands indicate the same behaviour with only monotonic increase of the beamsize with wavelength in the longwave end of the bands. Against diffraction, a broadening of the beam pattern occurs when wavelength decreases in the shortwave regions, well pronounced in the larger bandwidth SLW. Not being due to aberrations otherwise strong appearance in longwave end of SSW should be seen, reduction of spatial coherence appears then as a good candidate.
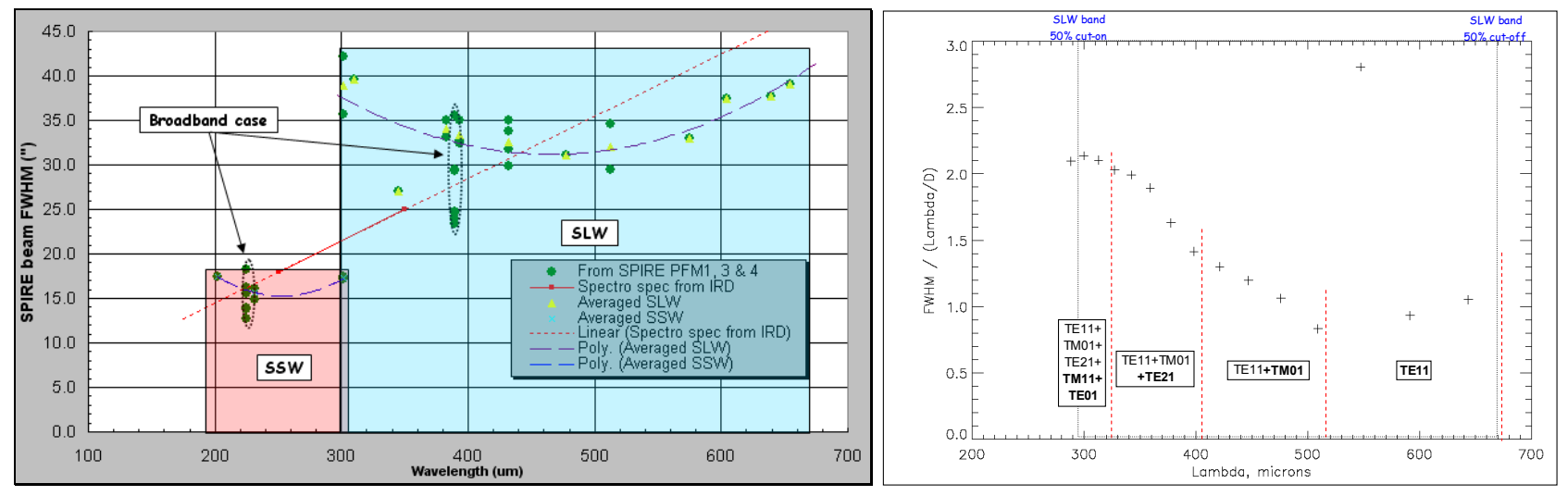

Figure 6: Spectrometer measured spectral beam size variations (left) and normalized to diffraction SLW beam size in-band variations with the waveguide modes and associated cut-off spectral locations (right)

This was further investigated via complementary test in which FTS spectra of broadband source were gathered at all spatial sample point locations of the standard beam map. From this 3D data cube, beamsize were extracted at different frequencies and Figure 6 right plot displays the spectral variations of these beamsizes normalised by the optical diffraction limit $\lambda / \mathrm{D}$. Only above $\sim 515 \mu \mathrm{m}$ in the region where only a single waveguide mode $\left(\mathrm{TE}_{11}\right.$, well approximated by a Gaussian shape) exists, FWHM is $\sim \lambda / \mathrm{D}$ as predicted by standard diffraction. Below, the beamsize broadening increases steadily with increasing number of modes allowed by the SLW waveguide ${ }^{[3]}$ interfacing with the bolometer cavity; and higher number of modes translate into reduced spatial coherence. 
The derived etendue only follows asymptotically the $\lambda^{2}$ variations for longwave part of each band. Expecting the stronger dominance of higher order modes accepted by the feedhorn+waveguide structure, the spectral average of the inband etendue was estimated for SSW and SLW, see Figure 7 below. As predicted by theory ${ }^{[11]}$, their ratio was found to match exactly the ratio of the respective waveguide cross-section $\left(\mathrm{d}_{\mathrm{SLW}} / \mathrm{d}_{\mathrm{SSW}}\right)^{2}=(395.5 \mu \mathrm{m} / 190.5 \mu \mathrm{m})^{2}=4.3$. This was further verified by plotting the number of modes $\mathrm{N}=\mathrm{A} \Omega / \lambda^{2}$ versus the normalised waveguide radius. The linear fit with slope $\sim 2$, parallel to the one expected from theory of few moded horn couplers ${ }^{[11]}$, confirms the dependence of the retrieved etendue on the waveguide surface area. Unlike in the case of the Photometer bands more dominated by an "optical" behaviour, the mode acceptance and modal filtering of the feedhorn+waveguide over larger relative bandwidths indicate here a more "detector" dominated behaviour for the Spectrometer end-to-end response.
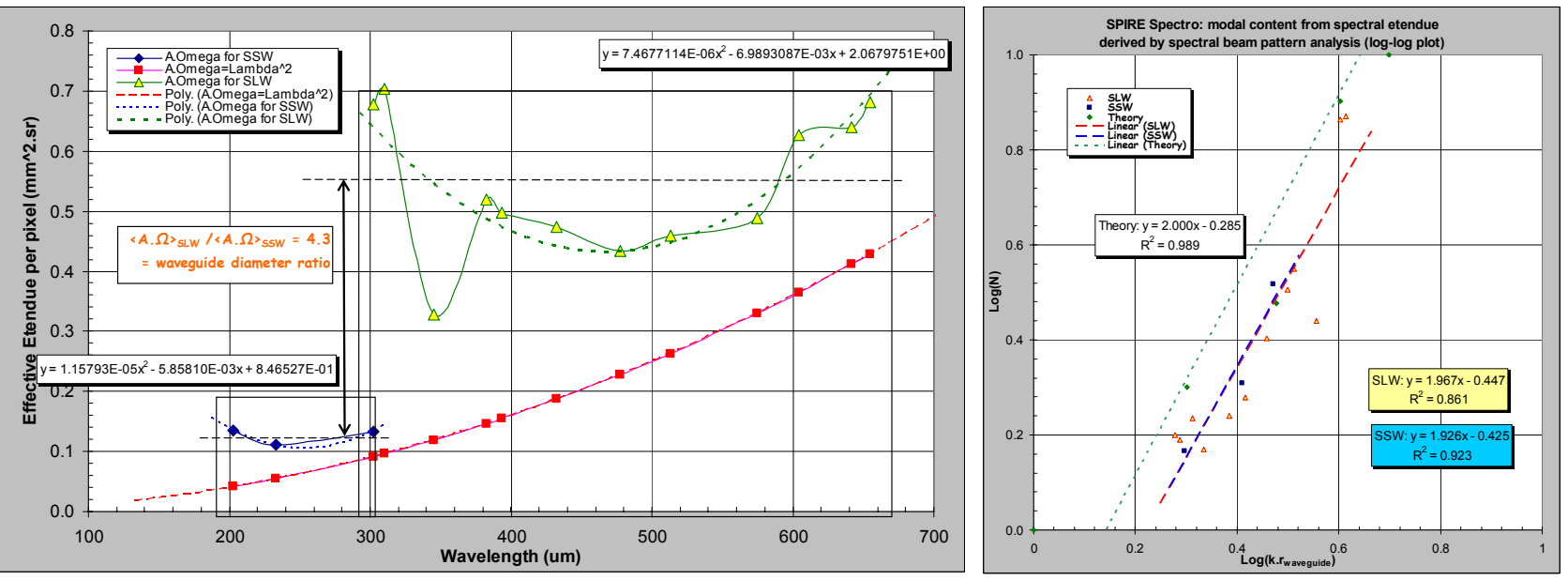

Figure 7: Retrieved Spectrometer etendue (left) and $\log -\log$ variations of the number of modes $N=A \Omega / \lambda^{2}$ with normalized waveguide radius $\mathrm{kr}_{\text {waveguide }}=2 \pi \mathrm{r}_{\text {waveguide }} / \lambda$ with comparison to waveguide theory (right)

As in the case of the Photometer, modal decomposition was performed on the spectral beam pattern data set and the relative power or fraction of fundamental mode is displayed in Figure 8. At the appearance of the $3^{\text {rd }}$ waveguide mode, this fraction is close to only $\sim 66 \%$ and drops further for SLW until at the shortwave edge of the band where it is no longer the dominant mode at all $(<50 \%)$. Below $330 \mu \mathrm{m}$, the spatial response of SLW is shaped by the higher order modes and is no longer maintaining a pure Gaussian profile. The higher order mode can enhance the overall coupling at short SLW in-band wavelengths on partially coherent sources but by participating to the etendue large values in the same spectral region, it can as well increase of accepted straylight by coupling to not-spatially-coherent background radiation.

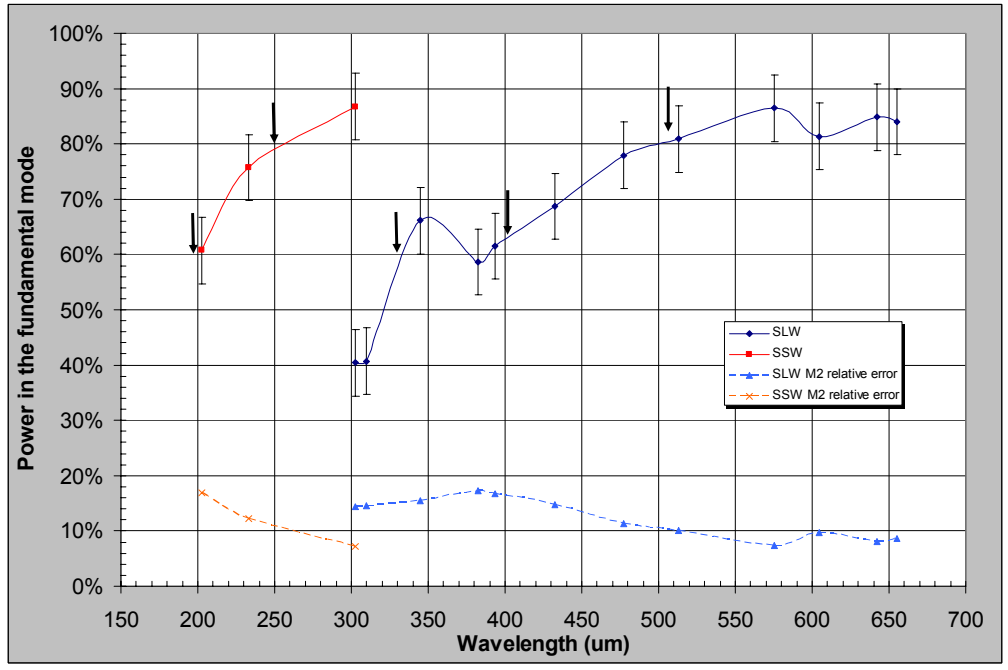

Figure 8: Spectral distribution of the fundamental mode relative power from the measured beam pattern modal decomposition in the Spectrometer bands. Additionally, black arrows indicate the spectral location of the respective mode cut-offs. 


\section{OTHER OPTICAL CHARACTERISTICS}

In the table below is summarised the final values for a set of optical parameters defined in the instrument requirements and subjected to test as part of the optical performances verification on-ground. They were derived mostly from series of 2D beamscans as well as peak-ups (made of 2 orthogonal cuts or 1D scans for faster mapping hence increase coverage of FoV) on large number of pixels over the different arrays.

\begin{tabular}{|c|c|c|}
\hline & PHOTOMETER & SPECTROMETER \\
\hline $\begin{array}{l}\text { Nominal final } \\
\text { focal ratio }\end{array}$ & $\begin{array}{l}\mathrm{F} \# \sim 4.9 \pm 0.25 \text { derived from tests, in agreement with } \\
\text { nominal design value }\end{array}$ & $\begin{array}{l}\mathrm{F} \#=4.85 \pm 0.15 \text { for } \mathrm{SLW} \text { and } 4.35 \pm 0.15 \text { for } \mathrm{SSW} \text {, in } \\
\text { agreement with nominal design values }\end{array}$ \\
\hline $\begin{array}{l}\text { Variation in } \\
\text { focal ratio }\end{array}$ & $< \pm 0.25$ or $5 \%$ max relative change & $\sim 5 \%$ max relative change across FoV \\
\hline Distortion & $\begin{array}{l}\text { Max level of }<8(+2 /-5) \% \text {, also in line with the } \\
\text { relative variation of } f \text {-number }\end{array}$ & $\begin{array}{l}\text { Max level of }<5 \% \text { also in-line with the relative } \\
\text { variation of } \mathrm{f} \text {-number }\end{array}$ \\
\hline Anamorphism & $\begin{array}{l}\text { Ellipticipty/anamorphism values of }<10 \% \text { for PLW, } \\
<15 \% \text { for PSW and } \sim 5 \% \text { for PMW. } \\
\text { Upper limits more often reach at FoV edge or } \\
\text { corners. Has been found higher when highly } \\
\text { (linear) polarised source such as FIR laser is used. }\end{array}$ & $\begin{array}{l}\text { Ellipticipty/anamorphism values of }<10 \% \text { for SLW } \\
\text { and SSW, on spatial response to broadband point } \\
\text { source. } \\
\text { Upper limits more often reach at FoV edge or } \\
\text { corners by diffraction at the edge of unvignetted } \\
\text { FoV. Has been found higher when highly (linear) } \\
\text { polarised source such as FIR laser is used. }\end{array}$ \\
\hline Image quality & $\begin{array}{l}\text { Strehl ratio }>99 \% \text { at } 250 \mathrm{um} \text { over full Phot FoV; } \\
\text { derived from measurement during STM warm } \\
\text { alignment }{ }^{[12]} \text {. } \\
\text { Derivation of in-band Strehl ratios from spectral } \\
\text { beam patterns measurements of spatial pixel } \\
\text { response and modelling of low Fresnel number } \\
\text { optical effect. Strehl values of } ~ 98 \pm 2 \% \text { for PSW, } \\
96 \pm 2 \% \text { for PMW and } 94 \pm 3 \% \text { for PLW; although in } \\
\text { highly diffraction-limited low Fresnel number } \\
\text { systems, Strehl is somewhat not adapted. } \\
\text { Apparent anomaly of the decrease with wavelength } \\
\text { explained by optical diffractive effect involved and } \\
\text { by measurements being performed at best focus for } \\
\text { individual wavelength. }\end{array}$ & $\begin{array}{l}\text { Strehl ratio }>95 \% \text { at } 250 \text { um at FoV centre; derived } \\
\text { from measurement during STM alignment }{ }^{[12]} \text { and } \\
\text { improved to }>97 \% \text { after astigmatism correction in } \\
\text { Spectro optical train for PFM. } \\
\text { NB: model based retrieval as in Photometer case } \\
\text { less adapted/accurate here due to overlapping of } \\
\text { detector modal behaviour i.e. pixel spatial response } \\
\text { no longer dominated by diffractive optical } \\
\text { behaviour but also modal/coherence effect from } \\
\text { detector }\end{array}$ \\
\hline Co-alignment & $\begin{array}{l}\text { Co-alignment between PLW and PMW is found } \\
<2 \pm 1 \text { arcsec, co-alignment between PSW and PMW } \\
\text { is found }<4 \pm 2.5 \text { arcsec. }\end{array}$ & $\begin{array}{l}<1.5 \pm 1 \text { arcsec, applicable only to the pixels at FoV } \\
\text { centre as these are now the only ones by design to } \\
\text { be nominally co-aligned. }\end{array}$ \\
\hline
\end{tabular}

These optical characteristics have been found compliant with the optics-related instrument requirements, giving confidence in SPIRE flight model being able to meet its scientific goals, although further post-launch verifications are planned and will include beam pattern measurements (with the use of the SPIRE BSM) and re-derivation of some of the above characteristics. 


\section{FURTHER OPTICAL CHARACTERISATION}

Beyond the verification of the nominally required optical performances of SPIRE, series of specific tests were developed and implemented to allow further characterisation in particular of relevant aspects to overall optical instrument performances while not being easily or precisely measurable in orbit.

\subsection{Defocus effect}

The broadband test beam is pointed at co-aligned pixels on Spectrometer and Photometer channels respectively and for a series of axial position creating a defocus at the instrument optical interface (the telescope focal surface, nominally confocal with SPIRE entrance focal plane), beamscans for the illuminated pixels are gathered.
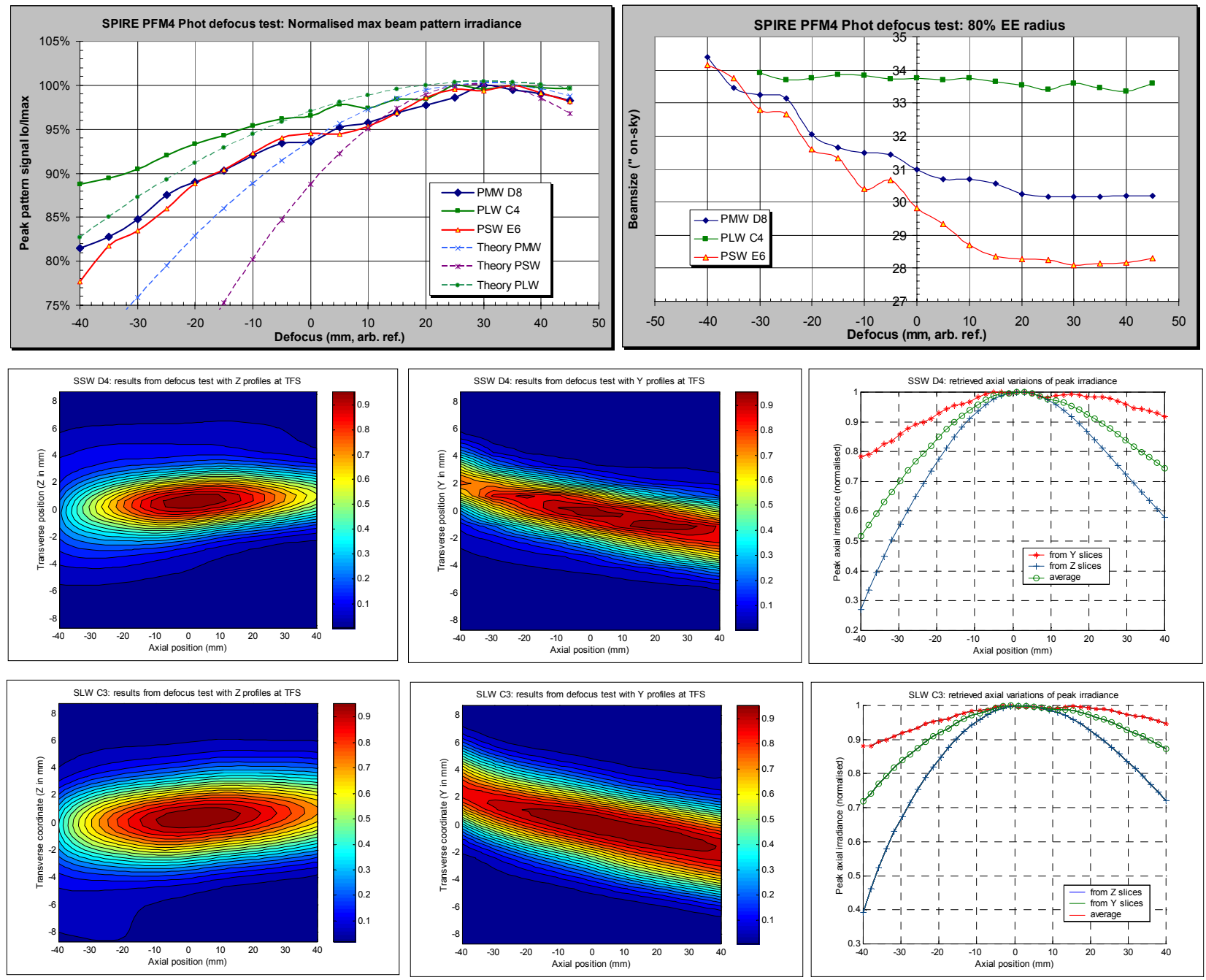

Figure 9: Results from the defocus test on the Photometer (top two plots) and Spectrometer (bottom six graphs).

The beamsize and peak irradiance variations with defocus follow the expected behaviour. The angular tilt or distortion seen in the Spectrometer axial data are due, as some residual astigmatism, to the test set-up no longer to perfectly maintain field and pupil at very large defocus (many $\mathrm{cm}$ ). In the case of the Photometer, a beam size criterion, taken here as the $80 \%$ EE radius of the measured beam pattern, indicates as expected the increase in depth-of-focus with increasing array central wavelength while the peak irradiances was compared to an analytic model of axial irradiance. Differences in the latter one are due to the effect test source finite extent (not perfect point source for SNR reason at large defocus), explaining why the matching is better for larger pixel compared to source size geometric image, i.e. PLW with matching over $\sim 40 \mathrm{~mm}$ defocus while only $\sim 30$ and $\sim 20 \mathrm{~mm}$ for PMW and PSW respectively. 


\subsection{Source size effect}

Again, the broadband source is pointed at co-aligned pixels around Photometer and Spectrometer. The source diameter d is varied in size from sub-pixel size to larger than 1 pixel and spatial pixel response measured for each position $d$ via $2 \mathrm{D}$ beamscan; all other parameters being kept constant. The dependence of resulting quantities is expressed in reduced source size (area), via the dimensionless ratio $\left(d / d_{0}\right)^{2}$ where $d_{0}=2 F \lambda_{0}$ is the focal plane pixel size (feedhorn aperture) for each respective band.
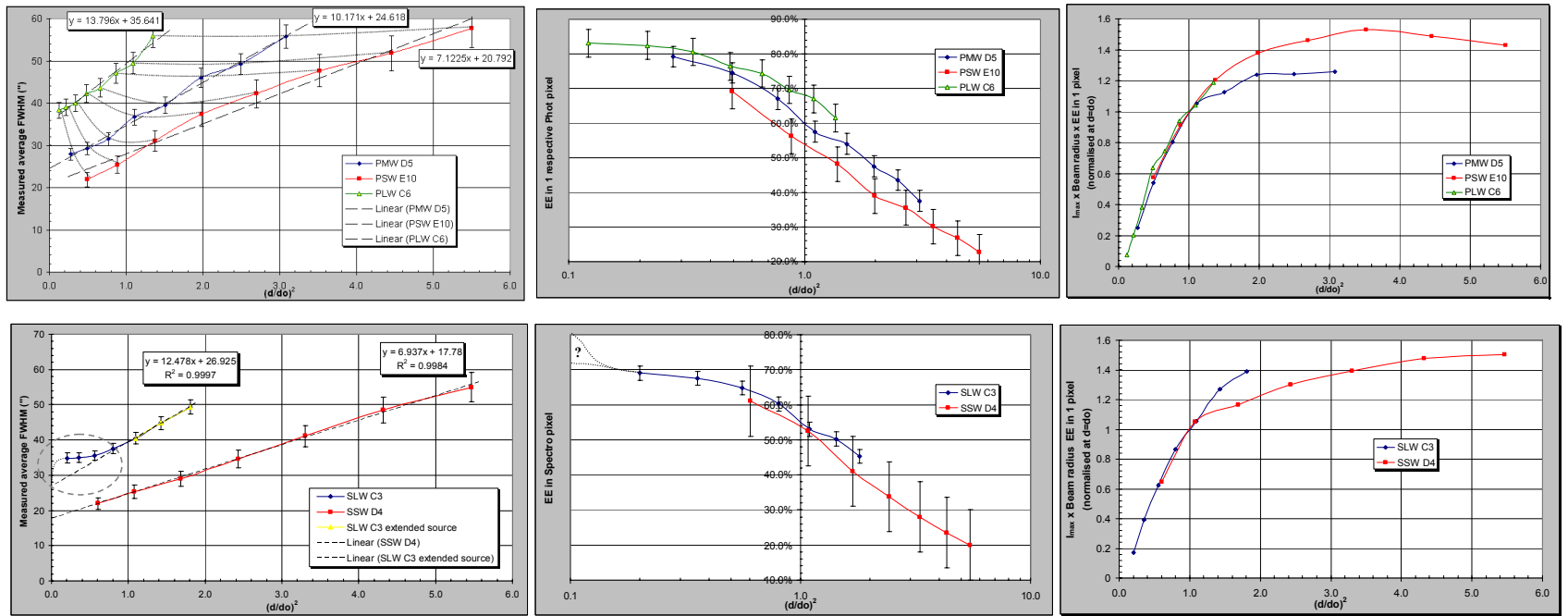

Figure 10: Beamsize, encircled energy inside a pixel aperture and total signal per pixel in each band as function of the reduced source size area for the Photometer (top) and the Spectrometer (bottom)

The total detected signal as function of source size is linked to standard aperture photometry ${ }^{[13]}$ and here the linear behaviour for all bands is found when source extent is up to the respective band pixel size. The beamsize variations follow an expected pattern in general. The extrapolated y-axis intercept (i.e. the point source case) on the linear fit to beamsize variations provides a direct alternative to the retrieval of SPIRE instrumental spatial response without the (de)convolution of the source size and is found in good agreement with the other methods, only slight modulated in the case of co-aligned pixels by the focus optimization for one of 3 co-aligned pixels and therefore bands. The encircled energy (EE) reaches a plateau around $\sim 80 \pm 5 \%$ typically for $\mathrm{d} \leq \lambda_{\mathrm{o}} / \mathrm{D}$, consistent of a standard optical behaviour of the Photometer bands. For the Spectrometer, a slightly anomalous spatial response for quasi-point source (continuum spectrum) with size from $\sim \lambda_{\mathrm{o}} / 3 \mathrm{D}$ to $\sim 3 \lambda_{\mathrm{o}} / 2 \mathrm{D}$ is found and seems linked to the lower than expected plateau for same source size range in the EE values. It has been found experimentally difficult due to low SNR to probe the very small source size region but scattered values of beamsize from 25 " to 35 " have been found for SLW with broadband thermal source. This could be linked to the dominant multi-modal behaviour over the broad SLW band and therefore caution should be applied when pointing, with SLW array, at continuum (e.g. thermal RJ source but ultimately source spectrumdependent as well) source with scale at the edge or below the optical resolution $\lambda_{\mathrm{o}} / \mathrm{D}$.

\subsection{Instrumental polarisation}

SPIRE not being a polarimeter, polarisation states of the detected photons are not intended to be measured in-flight. Nevertheless, residual instrumental polarisation can affect the accuracy of the retrieved source photometry. Here a rotating wire-grid polariser was inserted in the broadband test source path (HBB) while pointing at the test beam at sets co-aligned Photometer and Spectrometer pixels. The signal s for each pixel in each band is recorded as function of the polariser angle $\theta$ and then fitted with a $\cos ^{2}\left(\theta+\phi_{0}\right)$ model. The instrumental polarisation rate $p$ is then obtained as $\mathrm{p}=\left(\mathrm{s}_{\max }-\mathrm{s}_{\min }\right) /\left(\mathrm{s}_{\max }+\mathrm{s}_{\min }\right)$. The test was repeated for reproducibility and comparison is given below with simulations with an optical raytrace model of the instrument and test configuration using inputs from the previously measured in-band average transmission and reflection values of components such as filters, dichroics and beamsplitters. 

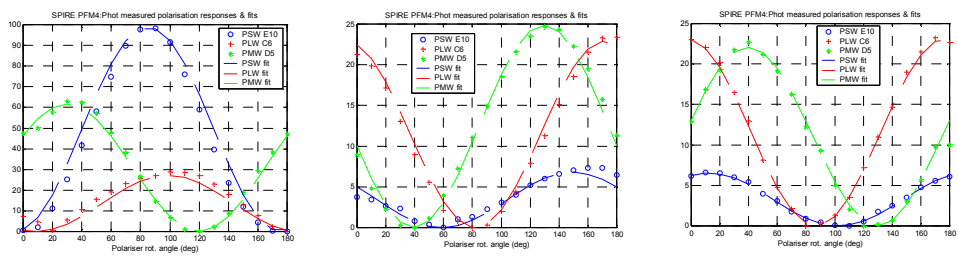

\begin{tabular}{|l|c|c|c|}
\hline & PSW & PMW & PLW \\
\hline Pol. rate p (PFM4) & $6.0+/-1.2 \%$ & $10.6+/-2.5 \%$ & $9.5+/-5.5 \%$ \\
\hline Optical model & $5.7+l-1.3 \%$ & $7.6+l-1.1 \%$ & $5.4+/-1.5 \%$ \\
\hline
\end{tabular}
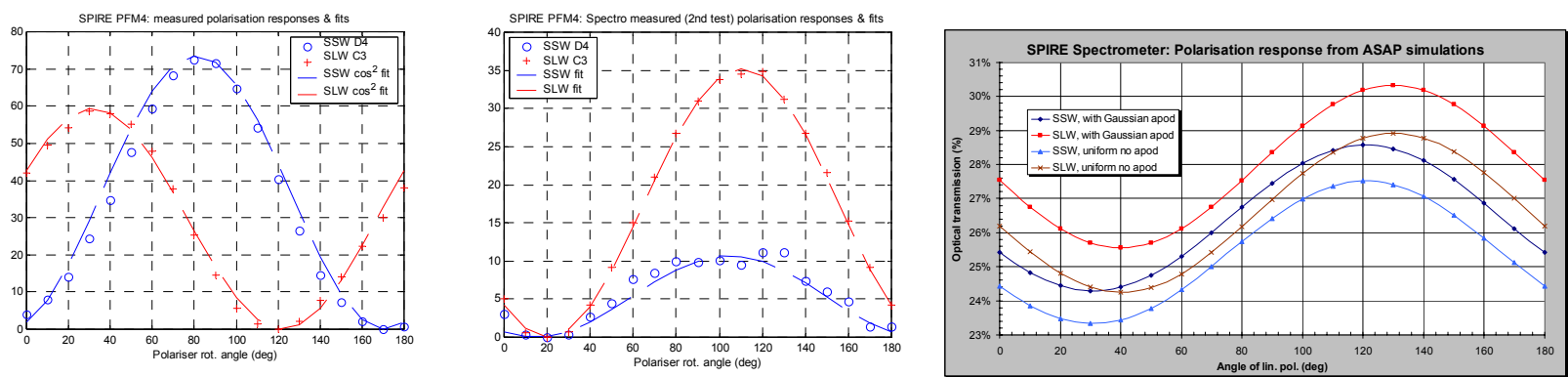

Figure 11: Measured optical signal variations for each pixel/array as function of linear polariser angle and associated fits: case of the Photometer (top) and Spectrometer (bottom); on the right side of each case, comparison with results from optical model predictions.

In the first test configuration, to avoid standing waves, the polariser was tilted but this increased the apparent wire grid pitch leading to a more grating-like behaviour in the shortwave bands (PSW and SSW). The polariser was then moved to a pupil plane location normal to the test beam. This led to a good match of the relative phases in the Spectrometer case, where a relative phase shift is expected even in a symmetric optics interferometer configuration due to the output arms (or bands here) looking at RR \& TT and RT \& TR beamsplitter characteristics respectively. An experimental polarisation rate of $\sim 7.3 \%$ for SLW FoV centre compares approximately well with the model predictions of $\sim 8.7 \%$. There is reasonable agreement for the Photometer arrays case, although the model does not take into account the inherent effect of the quasi-optical components structure ${ }^{[14]}$, and overall these results give a reasonable idea of the amount of photometric signal variation to be expected per pixel from polarised source and by comparison to unpolarised ones.

\section{SOME RESULTS OF SPIRE FLIGHT SPARE MODEL OPTICAL TESTING}

The Flight Spare (FS) model of SPIRE went through operating temperature performance verification. This test campaign, completed less than 2 months ago, included optical characterisation tests as performed on the flight model. And we are pleased to be able to report here, some of the preliminary results from the processing of the measured data. In particular, the measured beam patterns, via 2D beam map, on different pixels of all arrays indicated good quality shape as illustrated below and reproducibility across respective array.
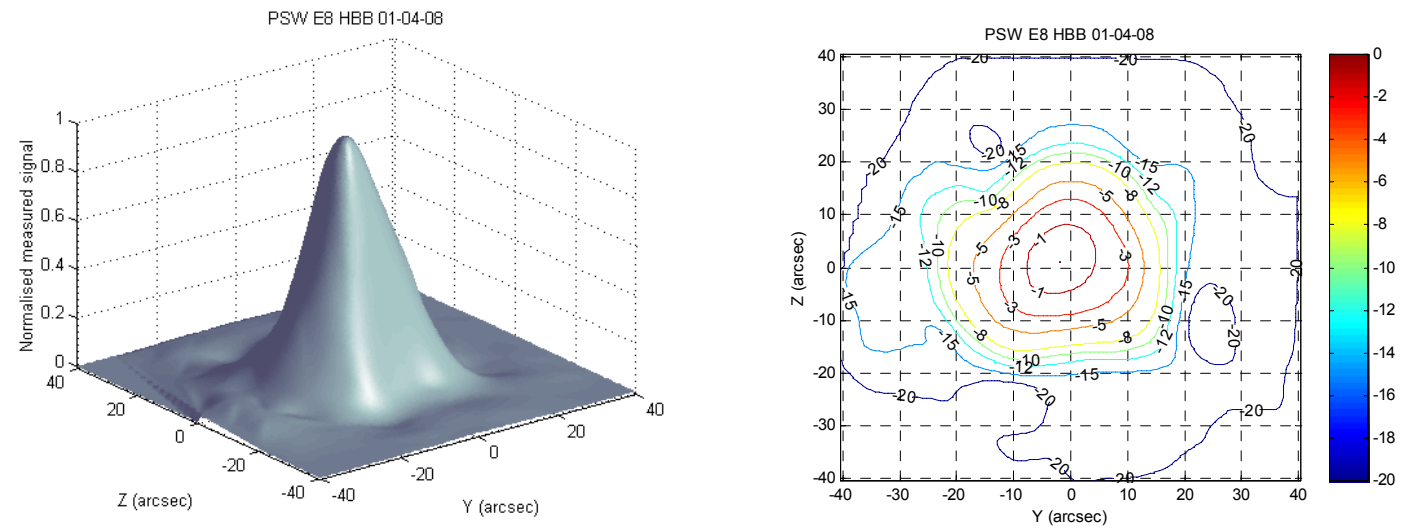

Figure 12: Measured beam pattern for the FS Photometer reference pixel (i.e. PSW E8 at Photometer FoV centre) with a quasi point source and broadband spectrum (HBB): 3D and linear scale (left), log scale contour plot (right) 
The response to different source size extents was also probed on Photometer and Spectrometer respectively co-aligned pixels and the resulting behaviour was found satisfactory, comparing well with flight model results (see section 5.2).
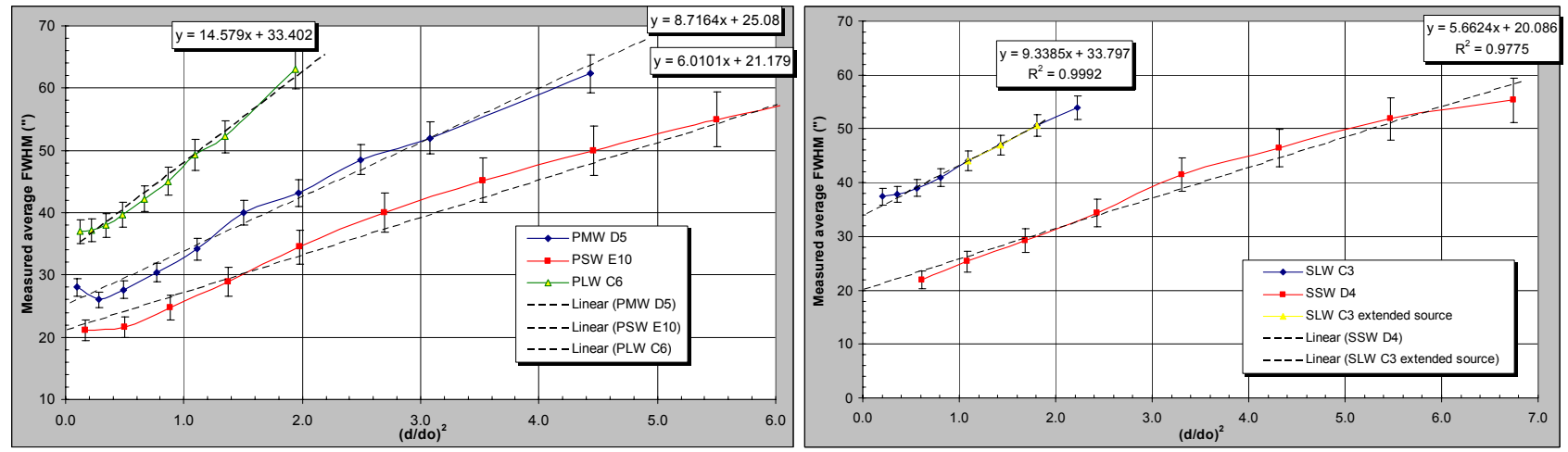

Figure 13: Variations of the measured beam pattern size (FWHM) as function of the relative source size area for FS Photometer arrays (left) and Spectrometer arrays (right). For comparison with FM similar data in Figure 10.

And finally instrumental polarisation level was measured with the same procedure as for the flight model and results for the Photometer are given below. The test was actually performed twice on 2 different sets of co-aligned pixels to probe eventual field angle dependence but actually no clear dependence on FoV position was found, i.e. measured differences were within measurement errors.
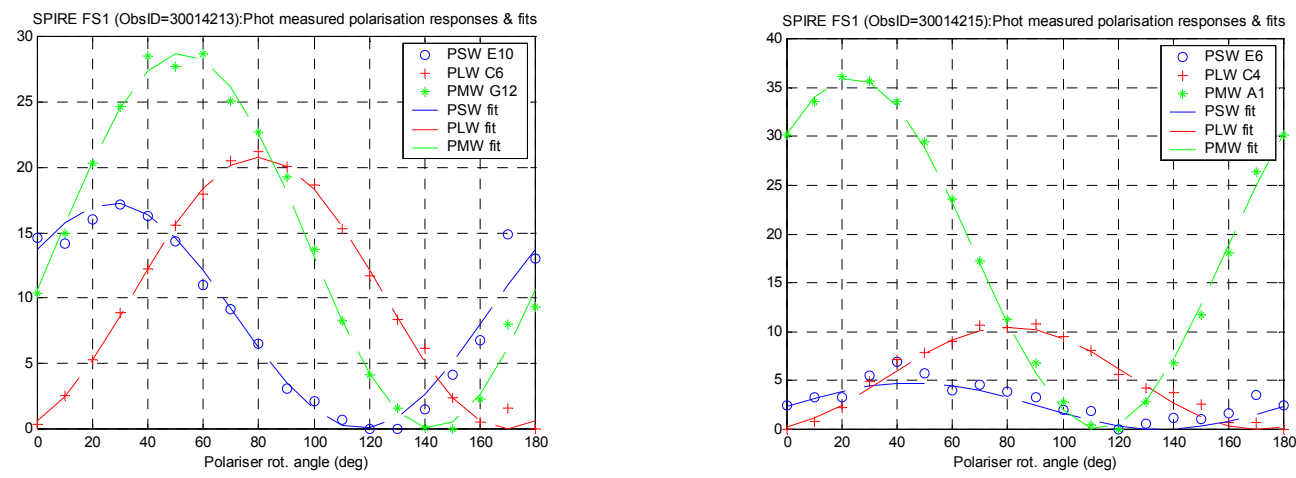

\begin{tabular}{|l|c|c|c|}
\hline & PSW E10 & PMW G12 & PLW C6 \\
\hline Polarisation rate p & $4.3 \%$ & $6.8 \%$ & $6.2 \%$ \\
\hline Phase $\left(\right.$ in cos $^{2}$ ) & $-27 \mathrm{deg}$ & $-52.5 \mathrm{deg}$ & $-79.8 \mathrm{deg}$ \\
\hline & PSW E6 & PMW A1 & PLW C4 \\
\hline Polarisation rate p & $2.6 \%$ & $6.8 \%$ & $4.6 \%$ \\
\hline Phase (in $\cos ^{2}$ ) & $-45.5 \mathrm{deg}$ & $-23.5 \mathrm{deg}$ & $-80.8 \mathrm{deg}$ \\
\hline
\end{tabular}

Figure 14: Measured optical signal variations for each pixel/array as function of linear polariser angle and associated fits (top) and summary of the derived polarisation rates and relative phases for SPIRE FS Photometer (bottom). For comparison with Figure 11.

Even the relative phases (or phase differences) between arrays are found as expected from the geometry, including the constant one for PLW (= the array "in-plane") and swapping of PSW/PMW value due to symmetry along chop or Y axis about the FoV centre, of the chosen co-aligned ones. The polarisation rate in all bands is low and equal or lower than the optical model predictions indicating a low impact on photometry.

These first results indicate good optical performances and optical representativity of the SPIRE flight spare model. 


\section{ACKNOWLEDGEMENTS}

We acknowledge the support and collaboration with the entire SPIRE instrument consortium ${ }^{[1]}$ led by the Principal Investigators Matt Griffin (Cardiff University - UK) and Laurent Vigroux (IAP - France) and, through the SPIRE project management, by Ken King (RAL - UK) and Eric Sawyer (RAL - UK).

\section{REFERENCES}

[1] M. Griffin et al., "Herschel-SPIRE: design, ground test results, and predicted performance", Proc. SPIE 7010 (2008)

[2] B.M. Swinyard et al., "Imaging FTS for Herschel-SPIRE”, Proc. SPIE 4850 (2002)

[3] G. Chattopadhyay et al., "Feed Horn Coupled Bolometer Arrays for SPIRE: Design, Simulations, and Measurements", IEEE Trans. MTT 51-10 (2003)

[4] K. Dohlen et al., "Optical design of the SPIRE instrument for FIRST”, Proc. SPIE 4013 (2000)

[5] T.L. Lim et al., "Preliminary results from Herschel-SPIRE flight model testing”, Proc. SPIE 6265 (2006)

[6] B.M. Swinyard et al., "Optical performance characterisation of Herschel-SPIRE”, Proc. SPIE 6265 (2006)

[7] L.D. Spencer et al., "Performance evaluation of the Herschel-SPIRE instrument flight model imaging FTS", Proc. SPIE 7010 (2008)

[8] P. Collins et al., "Ground calibration facility for Herschel-SPIRE”, Proc. SPIE 4850 (2002)

[9] M. Ferlet, "Imaging at low Fresnel number: challenges and applications", Proc. SPIE 6289 (2006)

[10] D.H. Martin et al., "Long-wave optics”, IEEE Trans. MTT 41 (1993)

[11] J.A. Murphy et al., "Radiation patterns of few-moded horns and condensing lightpipes", Infrared Phys. 31-3 (1991)

[12] K. Dohlen et al., "Optical alignment verification of the Herschel-SPIRE instrument", Proc. SPIE (2004)

[13] P.A.R Ade et al., "The Queen Mary College/University of Oregon photometer for submillimetre continuum observations", Infrared Phys. 24-4 (1984)

[14] P.A.R. Ade et al., “A review of metal mesh filters”, Proc. SPIE 6275 (2006) 